TAO, Vol. 15, No. 4, 683-695, November 2004

\title{
Coseismic Movement and Seismic Ground Motion Associated with the 31 March 2002 off Hualien, Taiwan, Earthquake
}

\author{
Horng-Yue Chen ${ }^{1, *}$, Long-Chen Kuo ${ }^{1}$, and Shui-Beih Yu ${ }^{1}$
}

(Manuscript received 19 January 2004, in final form 5 October 2004)

\begin{abstract}
On 31 March 2002, a $M_{L}=6.8$ earthquake (called the "331 earthquake") occurred in northeastern Taiwan off Hualien. The shock lasted over 30 seconds across the entire island of Taiwan. The earthquake caused a few centimeters of coseismic deformation, and a few centimeters of ground motion in the northern and eastern parts of Taiwan. All of these displacements have been recorded by the Taiwan Continuous GPS Array (TCGA), and estimated by two different post-processing methods, namely the daily solution and the kinematic positioning algorithm. Precise evaluation of the coseismic deformation and capturing instantaneous ground motion at a level of just millimeters requires rigorous computational procedures. In this paper, a completely regular algorithm to estimate the crustal deformation in the Taiwan area has been applied to acquire coseismic deformation as a result of the 331 earthquake. A set of high sampling rate $(1 \mathrm{~Hz})$ data from the TCGA has been used to study simultaneous ground motion during the 331 earthquake. Using over 18 months of data and 100 stations of the TCGA, the coseismic deformation due to the 331 earthquake can be precisely estimated, and instantaneous ground motion can be observed in that portion of the TCGA network where stations with high sampling rate observations are situated. Since the coseismic deformation can be acquired precisely and the instantaneous ground motion can be computed by continuous GPS observations and integrated with seismic data, these results can aid the study of seismology and earthquake geodesy.
\end{abstract}

(Key words: Continuous GPS observation, TCGA, Coseismic deformation, Seismic ground motion)

\footnotetext{
${ }^{1}$ Institute of Earth Sciences, Academia Sinica, Taiwan, ROC

* Corresponding author address. Dr. Horng-Yue Chen, Institute of Earth Sciences, Academia Sinica, Taiwan, ROC; E-mail: chenhy@earth.sinica.edu.tw
} 


\section{INTRODUCTION}

For earthquake motion studies, pre-seismic deformation is often more important than postseismic displacement information. The challenge therefore is to detect precursor motion, with uniformly high accuracy, across a wide range of spatial scales (Mervart 1995; Yu et al. 1997; Kuo 2001). For example, because of the unpredictable characteristics of earthquakes such as that which occurred in Taiwan on 21 September 1999 (the Chi-Chi earthquake), with a magnitude of 7.3 on the Richter scale, and a death toll of more than 2000 people killed (Chien 1999), together with the sparsely distributed GPS stations within an active fault monitoring system, the efficiency of such monitoring systems is as important as other deformation monitoring systems.

After the Chi-Chi earthquake, the Institute of Earth Sciences, Academia Sinica (IESAS) reexamined station spacing, data recording, conservation and the type of transmission of the TCGA, which is mainly composed of IESAS, Central Weather Bureau (CWB), Ministry of the Interior (MOI), and other institutes. Using funding provided by the National Science Council (NSC), the IESAS cooperated with the CWB to relocate geodetic GPS receivers with high sampling rates, safety data storing devices and the facility of real-time transmission equipment. From 2000 to 2005 this initiative expanded the TCGA to a 200-station multifunction monitoring system with station spacing of less than $20 \mathrm{~km}$. The short-term task is to detect pre-seismic, coseismic and post-seismic movements. The long-term mission is to monitor plate boundary deformation.

During this period, a $\mathrm{M}_{\mathrm{L}}=6.8$ earthquake (331 earthquake) occurred in the northeastern part of Taiwan, off Hualien on 31 March 2002. Data sets of two sampling rates (30 second and 1 second) were collected by TCGA. The coseismic deformation can be estimated by most of the stations storing the 30-second sampling rate data, and the instantaneous ground motion can be detected at the new stations with a $1 \mathrm{~Hz}$ logging rate. The basic strategy for estimating coseismic deformation is to compute relative positions using static mode, post-processing, and daily solution algorithm in combination with suitable processing models (Chen et al. 2003a). By analyzing the network solution of TCGA of more than 100 stations with 18 months of data ( 9 months before and 9 months after the earthquake) and applying a least-squares fitting algorithm, the coseismic deformation can be acquired precisely. For detection of instantaneous ground motion, we utilize the Quasi Ionosphere-Free (QIF) post-processing kinematic positioning method (Chen et al. 2003b) to estimate the real-time positioning for 7 stations in the TCGA over day 089 to day 091 2002. Using an outlier detection algorithm and filter processing for sidereal repeat noises (Bock et al. 2000), instantaneous ground motion can be determined in the epoch-by-epoch solutions.

\section{DATA ACQUISITION AND PROCESSING}

The design of a permanent GPS reference station network is dependent upon many factors, including the topography and geological characteristics of the region (in particular the location of tectonic faults). Therefore the inter-station distances may vary considerably. In the case 
of monitoring the pre-seismic or post-seismic displacement of a fault, a sub-kilometer resolution may be necessary (Rizos et al. 2000). A permanent GPS monitoring network was set up and has collected data in the Taiwan area since August 1992. Following the increment of the cooperative institutes and funded by the NSC, IESAS and other projects, until December 2002 the TCGA is composed of more than 100 stations (Fig. 1), and the data collecting, processing, and analyzing has been operated by IESAS regularly (Kuo 2001).

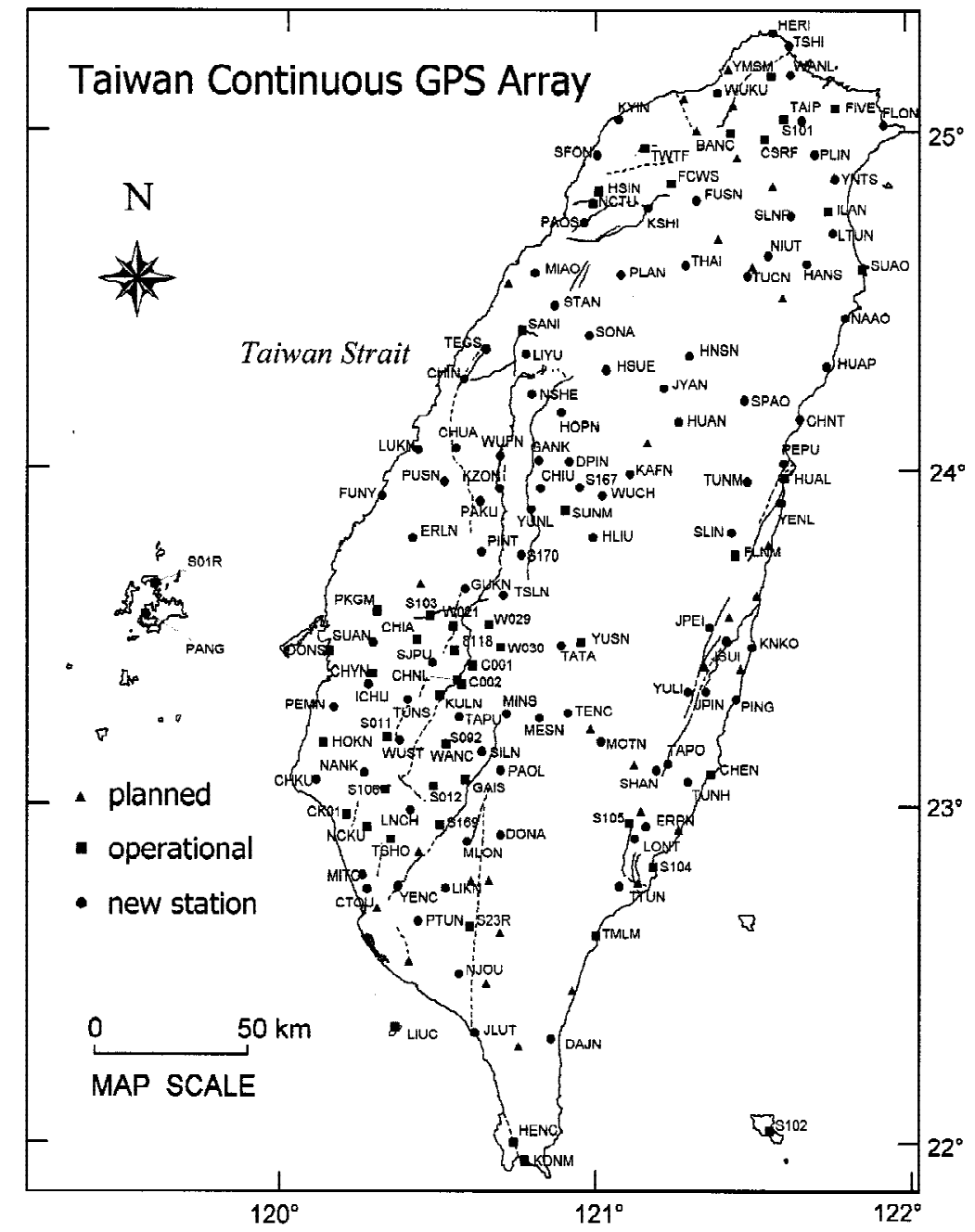

Fig. 1. Taiwan Continuous GPS Array: squares denote pre-existing operational stations, circles indicate new stations established after 1999 Chi-Chi earthquake, and triangles are planned stations. The active faults published by Central Geological Survey are also shown by curved solid or dash lines. 
There are two types of data logging at the newly setup stations of the TCGA, namely 1 second $(1 \mathrm{~Hz})$ and 30 second sampling rates. The daily solution uses the data with a 30 second sampling rate and can be used for determining plate boundary movement and coseismic deformation after a long time period. Once the earthquake occurred, $1 \mathrm{~Hz}$ data is stored in a ring buffer with up to 48 hours safety storage space. This high rate data can be transferred to the recording center of the IESAS to monitor ground motion during an earthquake. After 2005, most of the stations within the TCGA, will switch to modern receivers that can record different data logging rates to accommodate multifunction applications.

After careful selection of the TCGA sites, and using both hardware and software multipath error reduction techniques, the effect of multi-path error can be assumed to have been significantly mitigated (Han and Rizos 2000). For the orbit errors, the precise ephemerides from the International GPS service (IGS) were used and fixed in the data processing. Hence, the main biases in precise positioning are the ionospheric and tropospheric delays. The ambiguities and the coordinates of the TCGA stations, together with the parameters of the ionospheric delay and tropospheric delay, can be estimated from several hours of observation data. In all cases, data processing of the TCGA is conducted using Bernese software V. 4.2. For daily solutions using 30-second data from the TCGA stations, the Bernese program ADDNEQ, which can form an integrated daily solution with a consistent reference frame, is used. For the ground motion monitoring application with $1 \mathrm{~Hz}$ sampling rate, an outlier detection algorithm and a filtering process for sidereal repeat noises was applied to remove the system biases, yielding the simultaneous ground motion.

\subsection{Daily Solution for Estimating the Coseismic Deformation from TCGA}

Because the 331 earthquake occurred at UTC $06^{\mathrm{h}}: 52^{\mathrm{m}}: 54^{\mathrm{s}}$ (CWB earthquake report 2002), the daily solution of 31 March ignores the data before the time of the earthquake, and computes the rest of the data to be the first day of the post-seismic solutions. Through a careful processing procedure, the coseismic deformation can be obtained using the daily solution. Our solutions are tied to the coordinates and velocities of four global IGS fiducial stations, TSKB, SHAO, WUHN, TWTF, which are on the International Terrestrial Reference Frame 2000 (ITRF00). Eighteen months of daily solutions and more than 100 stations of the TCGA were analyzed in this study, which includes nine months before and after the 331 earthquake, respectively. Figure 2 shows the relative displacement at station SUAO with respect to S01R in the eighteen months from July 2001 to December 2002, which includes the pre-seismic, coseismic, and post-seismic displacements.

From Fig. 2, before the 331 earthquake (pre-seismic period, from year 2001.50 to 2002.25), the velocity of the northern component is $-18.8 \mathrm{~mm} \mathrm{yr}^{-1}$ and $15.4 \mathrm{~mm} \mathrm{yr}^{-1}$ for the eastern component. After a half year the velocity of the northern component becomes $-37.1 \mathrm{~mm} \mathrm{yr}^{-1}$ and $14.8 \mathrm{~mm} \mathrm{yr}^{-1}$ for the eastern component respectively, and the coseismic displacements are $-52 \mathrm{~mm}$ and $+18 \mathrm{~mm}$ in the north and east respectively. Significant transient post-seismic deformation in the horizontal components was also observed. It is obvious that the horizontal velocity becomes faster right after the 331 earthquake, and after a half year the horizontal velocity becomes slower. 


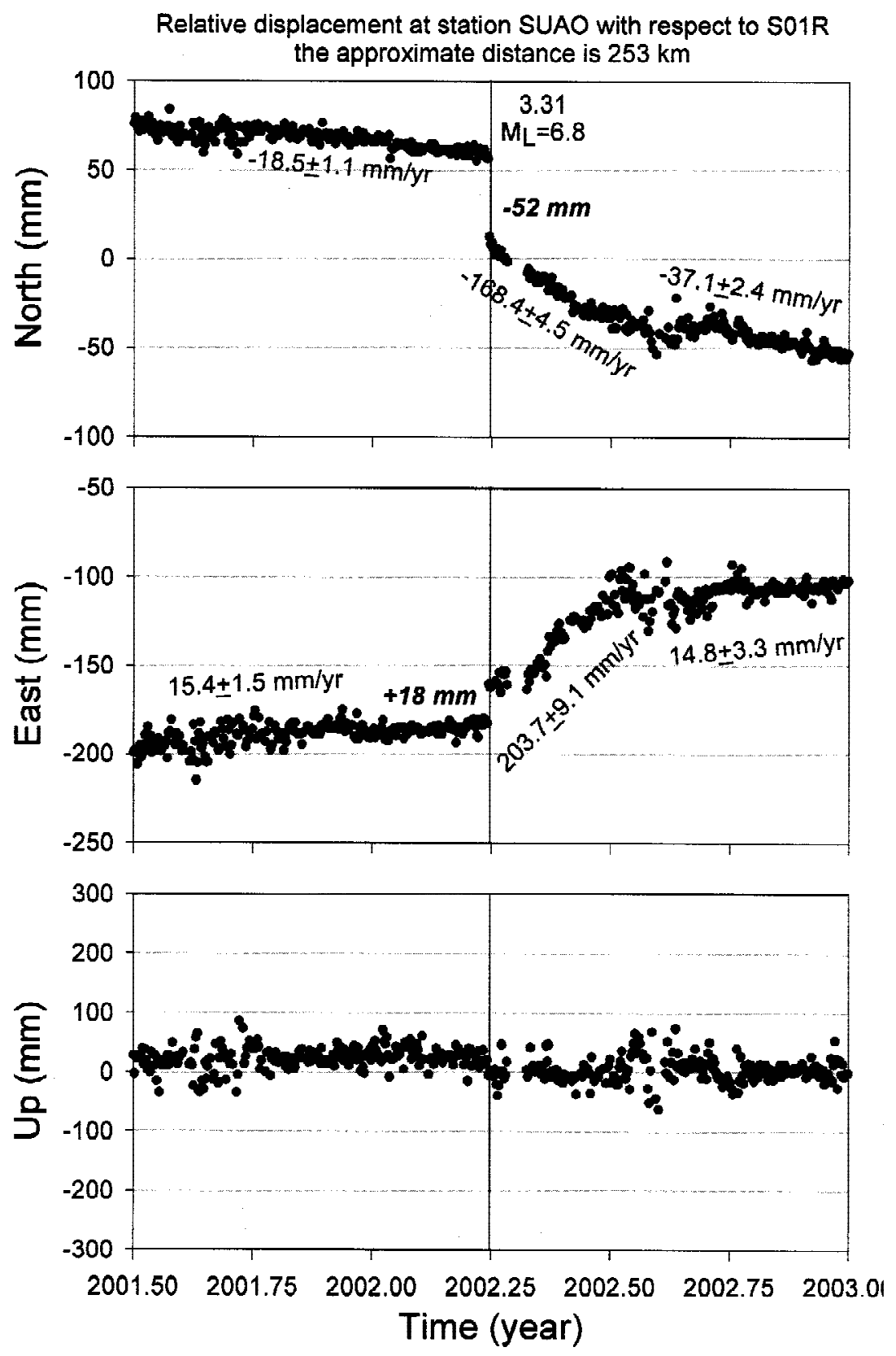

Fig. 2. The relative displacement at station SUAO. The abscissa denotes the time in years, and the ordinate represents the northern, eastern, and up components in $\mathrm{mm}$. 
A linear least-squares fitting algorithm is applied to detect outliers of the daily solutions in the north, east, and up components with time series before and after the earthquake, respectively. Subtracting the results from the linear fittings, the coseismic deformations can be obtained. Interactively comparing with the daily solution during the period from 2002.5 to 2002.75 , the results show a large scatter due to seasonal errors which may not be removed completely. Therefore, the practical findings of the daily solutions should be restricted to only three months before and after the 331 earthquake. Because some stations were far from the epicenter, the coseismic deformation is not significant at these points, and as a result only data from 40 stations of TCGA were used in this study. Although the GPS observations can obtain results in the north, east, and up components, the up components are rarely affected by the earthquake in this study due to longer distance from the epicenter. The horizontal displacements coseismic are shown in Table 1.

From the Table 1, the significant coseismic displacements of $14-55 \mathrm{~mm}$ in the $131^{\circ}$ $168^{\circ}$ directions appear in the northern part of the TCGA which are YMSM, FIVE, S101, BANC, ILAN, 0499 and SAUO. Due to the proximity of the epicenter (approximate $50 \mathrm{~km}$ ), the largest coseismic deformation emerges at station SAUO, and reaches $55 \mathrm{~mm}$. Relative to the southwestern stations from the epicenter namely PEPU, HUAL and TUNM, the coseismic deformation is only a few millimeters. The standard deviations of the north-south component are $3-4 \mathrm{~mm}$, and $4-5 \mathrm{~mm}$ to the east-west component, hence the accuracy of the north-south component is slightly better than the east-west component. All of the coseismic displacements with their 95\% error ellipses are plotted in Fig. 3.

Most of the larger coseismic displacements located in the northern part of the TCGA are oriented in a $160^{\circ}-170^{\circ}$ direction. The stations in the eastern margin of the Central Range (PEPU, TUNM, SLIN, FLNM, JPIN, and YULI), and the Coastal Range (HUAL, CHEN, and S104) only indicate horizontal displacements of $6.0-8.9 \mathrm{~mm}$, in a direction of $325^{\circ}-350^{\circ}$. The interface of the directions of the horizontal displacements is near the parallel of latitude at $24^{\circ} \mathrm{N}$.

\subsection{Kinematic Positioning Results to Detect Seismic Ground Motion}

To detect pre-seismic, coseismic and post-seismic deformations and study the crustal deformation of the plate boundary zone in and around Taiwan, the IESAS cooperated with the CWB to install geodetic GPS receivers with high sampling rate capability, safe data storing devices and real-time transmission equipment. With the funding of the National Science Council (NSC), this dense augmentation of the TCGA has been instituted for the period between 2000 and 2005 and resulted in a station spacing of $20 \mathrm{~km}$ and a 200 station multifunction monitoring system.

There are seven stations (S101, SLIN, PEPU, PLAN, NSHE, PINT, TSHO) of the TCGA running at a $1 \mathrm{~Hz}$ data sampling rate, which recorded the instantaneous seismic ground motion of the Hualien 331 earthquake. Due to the improvement of positioning algorithms, the accuracy of the medium-range kinematic relative positioning can reach centimeter levels (Bock et al. 2000; Remondi and Brown 2000). However, in the Taiwan area atmospheric conditions change dramatically and for the purpose of earthquake monitoring, the relative distance needs 
Table 1. Horizontal coseismic deformation with respect to Paisha, Penghu (S01R), for part of the TCGA GPS stations.

\begin{tabular}{|c|c|c|c|c|c|c|}
\hline Stations & $\begin{array}{c}\text { Lat. } \\
\left({ }^{\circ}\right)\end{array}$ & $\begin{array}{c}\text { Lon. } \\
\left({ }^{\circ}\right)\end{array}$ & $\begin{array}{l}\text { North } \\
(\mathrm{mm})\end{array}$ & $\begin{array}{c}\text { East } \\
(\mathrm{mm}) \\
\end{array}$ & $\begin{array}{l}\text { Disp. } \\
\text { (mm) }\end{array}$ & $\begin{array}{c}\text { Azi. } \\
\left({ }^{\circ}\right)\end{array}$ \\
\hline 0499 & 24.4560 & 122.9346 & $-10.4+3.3$ & $11.8 \pm 5.5$ & $15.7 \pm 6.4$ & 131.4 \\
\hline 0500 & 24.4282 & 123.7838 & $-1.5 \pm 3.5$ & $3.6 \pm 6.2$ & $3.6 \pm 7.1$ & 112.6 \\
\hline 0750 & 24.3408 & 124.1644 & $-1.2 \pm 3.2$ & $2.3 \pm 5.8$ & $2.6 \pm 6.6$ & 117.6 \\
\hline 0751 & 24.0634 & 123.7876 & $-6.5 \pm 3.5$ & $3.6 \pm 6.5$ & $7.4 \pm 7.4$ & 151.0 \\
\hline $\mathrm{BANC}$ & 24.9994 & 121.4338 & $-13.4 \pm 3.8$ & $4.5 \pm 4.7$ & $14.1 \pm 6.1$ & 161.4 \\
\hline CHEN & 23.0992 & 121.3654 & $5.8 \pm 3.1$ & $-1.0 \pm 4.4$ & $5.9 \pm 5.4$ & 350.2 \\
\hline FCWS & 24.8524 & 121.2416 & $-4.1 \pm 3.4$ & $2.1 \pm 4.5$ & $4.6 \pm 5.6$ & 152.9 \\
\hline FIVE & 25.0728 & 121.7728 & $-29.5 \pm 3.6$ & $7.0 \pm 4.1$ & $30.3 \pm 5.4$ & 166.7 \\
\hline FLNM & 23.7481 & 121.4452 & $7.6 \pm 3.5$ & $-1.4 \pm 4.8$ & $7.7 \pm 5.9$ & 349.6 \\
\hline GANK & 24.0301 & 120.8144 & $2.3 \pm 3.0$ & $-2.5 \pm 3.9$ & $3.4 \pm 4.9$ & 312.6 \\
\hline HNSN & 24.3394 & 121.2998 & $0.3 \pm 4.1$ & $-2.9 \pm 4.7$ & $2.9 \pm 6.2$ & 275.9 \\
\hline HOPN & 24.1725 & 120.8867 & $2.9 \pm 3.2$ & $-3.6 \pm 4.2$ & $4.6 \pm 5.2$ & 308.9 \\
\hline HSIN & 24.8295 & 121.0060 & $-0.8 \pm 3.6$ & $-0.4 \pm 4.3$ & $0.9 \pm 5.6$ & 206.6 \\
\hline HUAL & 23.9771 & 121.6053 & $8.2 \pm 3.6$ & $-3.4 \pm 4.7$ & $8.9 \pm 6.0$ & 337.5 \\
\hline HUAN & 24.1452 & 121.2644 & $3.8 \pm 3.9$ & $-11.4 \pm 4.8$ & $12.1 \pm 6.2$ & 288.4 \\
\hline ILAN & 24.7658 & 121.7484 & $-36.1 \pm 2.8$ & $13.6 \pm 3.7$ & $38.6 \pm 4.6$ & 159.4 \\
\hline JPEI & 23.5334 & 121.3632 & $7.5 \pm 3.6$ & $-2.1 \pm 5.2$ & $7.8 \pm 6.4$ & 344.4 \\
\hline KAFN & 23.9893 & 121.1083 & $3.4 \pm 3.3$ & $-2.1 \pm 3.9$ & $4.0 \pm 5.1$ & 328.3 \\
\hline KSHI & 24.7784 & 121.1678 & $-3.4 \pm 3.6$ & $1.5 \pm 4.0$ & $3.7 \pm 5.4$ & 156.2 \\
\hline PEPU & 24.0196 & 121.6021 & $6.2 \pm 3.8$ & $-4.3 \pm 4.8$ & $7.5 \pm 6.2$ & 325.3 \\
\hline PING & 23.3212 & 121.4462 & $6.7 \pm 3.1$ & $-2.1 \pm 4.5$ & $7.0 \pm 5.5$ & 342.6 \\
\hline PLAN & 24.5807 & 121.0784 & $0.0 \pm 3.8$ & $-1.7 \pm 4.5$ & $1.7 \pm 5.9$ & 270.0 \\
\hline S101 & 25.0422 & 121.6056 & $-20.8 \pm 3.5$ & $8.2 \pm 4.2$ & $22.4 \pm 5.4$ & 158.5 \\
\hline
\end{tabular}




\begin{tabular}{ccccccc} 
S104 & 22.8225 & 121.1813 & $4.9 \pm 4.1$ & $-4.2 \pm 4.9$ & $6.5 \pm 6.5$ & 319.4 \\
S167 & 23.9561 & 120.9259 & $2.4 \pm 3.1$ & $-2.9 \pm 4.0$ & $3.8 \pm 5.1$ & 309.6 \\
S170 & 23.7477 & 120.7595 & $2.9 \pm 3.0$ & $-2.7 \pm 4.1$ & $4.0 \pm 5.1$ & 317.0 \\
SANI & 24.4161 & 120.7605 & $-0.4 \pm 2.5$ & $-2.4 \pm 3.8$ & $2.4 \pm 4.5$ & 260.5 \\
SAUO & 23.8136 & 121.4332 & $-52.0 \pm 3.6$ & $18.1 \pm 4.9$ & $55.1 \pm 6.1$ & 160.8 \\
SLIN & 24.3995 & 120.9776 & $8.4 \pm 4.2$ & $-1.9 \pm 5.0$ & $8.6 \pm 6.5$ & 347.3 \\
SONA & 24.5942 & 121.8588 & $0.4 \pm 2.7$ & $-0.9 \pm 3.7$ & $1.0 \pm 4.5$ & 294.0 \\
SUN1 & 23.8830 & 120.9002 & $2.0 \pm 3.0$ & $-1.6 \pm 3.7$ & $2.6 \pm 4.7$ & 321.3 \\
THAI & 24.6089 & 121.2874 & $-2.0 \pm 3.5$ & $0.6 \pm 3.9$ & $2.1 \pm 5.2$ & 163.3 \\
TNML & 24.7997 & 120.9791 & $-1.6 \pm 3.5$ & $-0.7 \pm 3.9$ & $1.7 \pm 5.1$ & 203.6 \\
TSHO & 22.9059 & 120.3437 & $1.9 \pm 3.0$ & $-1.1 \pm 3.4$ & $2.2 \pm 4.6$ & 329.9 \\
TUNM & 23.9670 & 121.4854 & $6.6 \pm 4.2$ & $-3.2 \pm 5.2$ & $7.3 \pm 6.7$ & 334.1 \\
W029 & 23.5425 & 120.6561 & $2.8 \pm 3.4$ & $-2.2 \pm 3.9$ & $3.6 \pm 5.1$ & 321.8 \\
W030 & 23.4758 & 120.6874 & $2.9 \pm 2.7$ & $-2.6 \pm 4.1$ & $3.9 \pm 4.9$ & 318.1 \\
YMSM & 25.1674 & 121.5658 & $-19.1 \pm 4.4$ & $3.9 \pm 4.2$ & $19.5 \pm 6.1$ & 168.5 \\
YULI & 23.3427 & 121.2930 & $5.9 \pm 3.2$ & $-1.0 \pm 4.9$ & $6.0 \pm 5.9$ & 350.4 \\
YUSN & 23.4890 & 120.9510 & $2.0 \pm 5.3$ & $4.8 \pm 5.2$ & $5.2 \pm 7.4$ & 67.4 \\
\hline & & & & & & \\
\hline
\end{tabular}

(Table 1. continued)

to be a few tens to hundred of kilometers (Bock et al. 2000). Therefore the instantaneous positioning results are only acceptable with data at a high sampling rate (Remondi and Brown 2000). The real-time positioning results are useful in the short period of the earthquake, but the QIF results perform better over the whole practical time span (Chen et al. 2003b). 


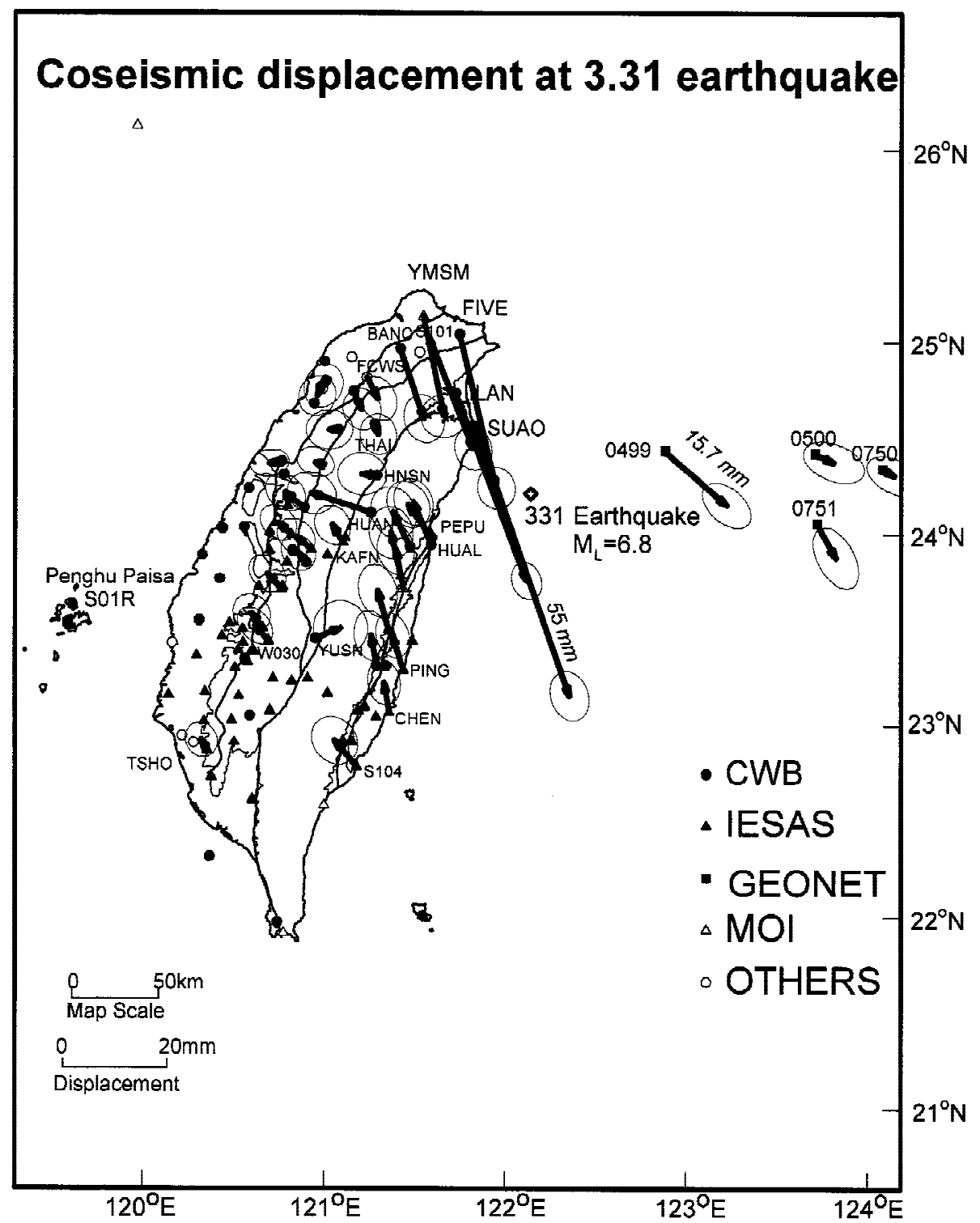

Fig. 3. The coseismic displacements of the 331 earthquake. The solid circles denote the CWB stations, the solid triangles represent the IESAS stations, the solid squares are the GEONET stations, the open triangles indicate the MOI stations, and the open circles show the stations from the other institutes. 
To obtain instantaneous ground motion, using the post-processing QIF algorithm to study the 331 earthquake, displacements with respect to station TSHO are computed. Based on relative positioning, displacement of the computing station with respect to the fixed station can be obtained at the arrival time, and the displacement of the fixed station can be obtained at the time of the second maximum ground motion. Hence the first maximum ground motion can be regarded as the maximum movement at the first station, and the second maximum ground motion can be treated as the maximum movement at the second station. The motion between the first and second maximum ground motion can be considered as the instantaneous ground motion at the first station. It is obvious that the coseismic ground motion can be observed using a $1 \mathrm{~Hz}$ sampling rate from continuous GPS stations.

Figure 4 shows the kinematic positioning results for station $\mathrm{S} 101$ with respect to stations NSHE (Fig. 4a) and THO (Fig. 4b), respectively. The first maximum ground motion occurred at $06^{\mathrm{h}}: 53^{\mathrm{m}}: 38^{\mathrm{s}}$ in both figures indicating the seismic motion at S101, while the second pulses denote the motion of the fixed stations, i.e., NSHE and TSHO.
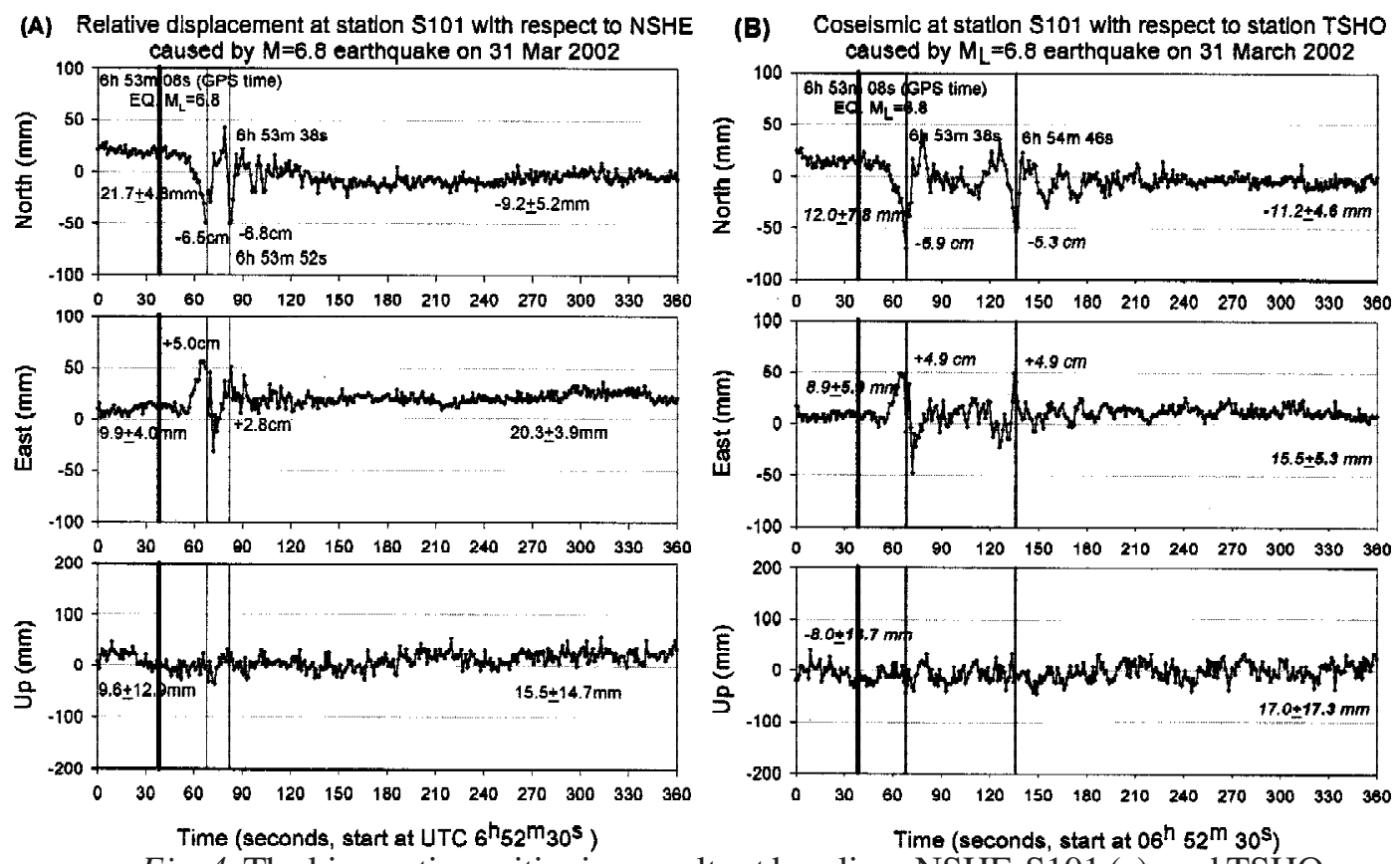

Fig. 4. The kinematic positioning results at baselines NSHE-S101 (a), and TSHOS101 (b) on 31 March 2002 (DoY, 090) with 1-second sampling rate. The abscissa denotes the time series, and ordinate represents the kinematic positioning results. 
The coseismic movement of station S101 is obtained from the mean values before and after the earthquake. Since the coseismic movement of station TSHO is not significant (Table 1), the relative displacement between $\mathrm{S} 101$ and TSHO can be taken as the coseismic displacement of S101. The coseismic displacements of S101 estimated from Fig. $4 \mathrm{~b}$ are $-23.2 \mathrm{~mm}$ and $+6.6 \mathrm{~mm}$ at the northern and eastern components, respectively. On the other hand, Table 1 shows the coseismic displacements of S101 from daily solutions are $-20.8 \mathrm{~mm}$ and $+8.2 \mathrm{~mm}$ at the northern and eastern components, respectively with the differences being only $2.5 \mathrm{~mm}$ and $1.6 \mathrm{~mm}$ at the northern and eastern components, respectively.

Because the arrival times at stations S101 and NSHE are too close (i.e., only 14 seconds in difference with time UTC: $06^{\mathrm{h}} 53^{\mathrm{m}} 38^{\mathrm{s}}$ and $06^{\mathrm{h}} 53^{\mathrm{m}} 52^{\mathrm{s}}$ ), the relative ground motion may be interfered. The maximum ground motion of station S101 in Fig. 4a is different from Fig. 4b for $4 \mathrm{~mm}$ and $1 \mathrm{~mm}$ to the north and east, respectively. But the arrival time at station S101 is almost the same. From Fig. 4 the seismic ground motion lasts over 80 seconds at $4 \mathrm{a}$, and 120 seconds at $4 \mathrm{~b}$. Hence, the farther the distance between two stations, the more detailed ground motion can be obtained.

Figure 5 shows the kinematic positioning results for 30-second, and 1-second sampling rates. The coseismic displacements of the 30 -second sampling rate data (Fig. 5a), and 1 -second sampling rate data (Fig. 5b) of the station S101 are also acquired from the mean values before and after the earthquake. Hence, the coseismic movement of the 30-second data at the northern component is $-19.1 \mathrm{~mm}$ and $4.6 \mathrm{~mm}$ at the eastern component. For the 1-second data, the coseismic movement at the northern component is $-23.2 \mathrm{~mm}$ and $6.6 \mathrm{~mm}$ at the eastern component. There are only a few millimeters difference between the results of the 30second sampling rate, and 1-second sampling rate. The results obtained from the 1-second sampling rate data can detect more detail of the ground motion, but the 30-second sampling rate data will smooth ground motion. Hence, the higher the data-sampling rate, the more detail the kinematic positioning results can obtain.

In this study, we just demonstrated the capability and precision of $1 \mathrm{~Hz}$ GPS data for detecting seismic ground motion. At this moment, the precision of high rate GPS measurement still needs to be improved. High rate GPS data just provide an independent measurement of ground motion and the GPS receivers may serve as geodetic seismometers in the future.

\section{CONCLUSION}

In this paper, the coseismic deformation of the Hualien, 331 earthquake in 2002 has been precisely obtained using daily solutions, and a comparison of the different sampling rates of the single epoch solutions are discussed. From these results the following conclusions can be drawn:

1. Despite an interim earthquake restricting the 18-month continuous data sequence, coseismic deformation can still be obtained from the TCGA using the three months before and after the 331 earthquake of the daily solutions. Because some stations were far from the epicenter, the coseismic deformation was not significant, and therefore only 40 stations participated in this study. 


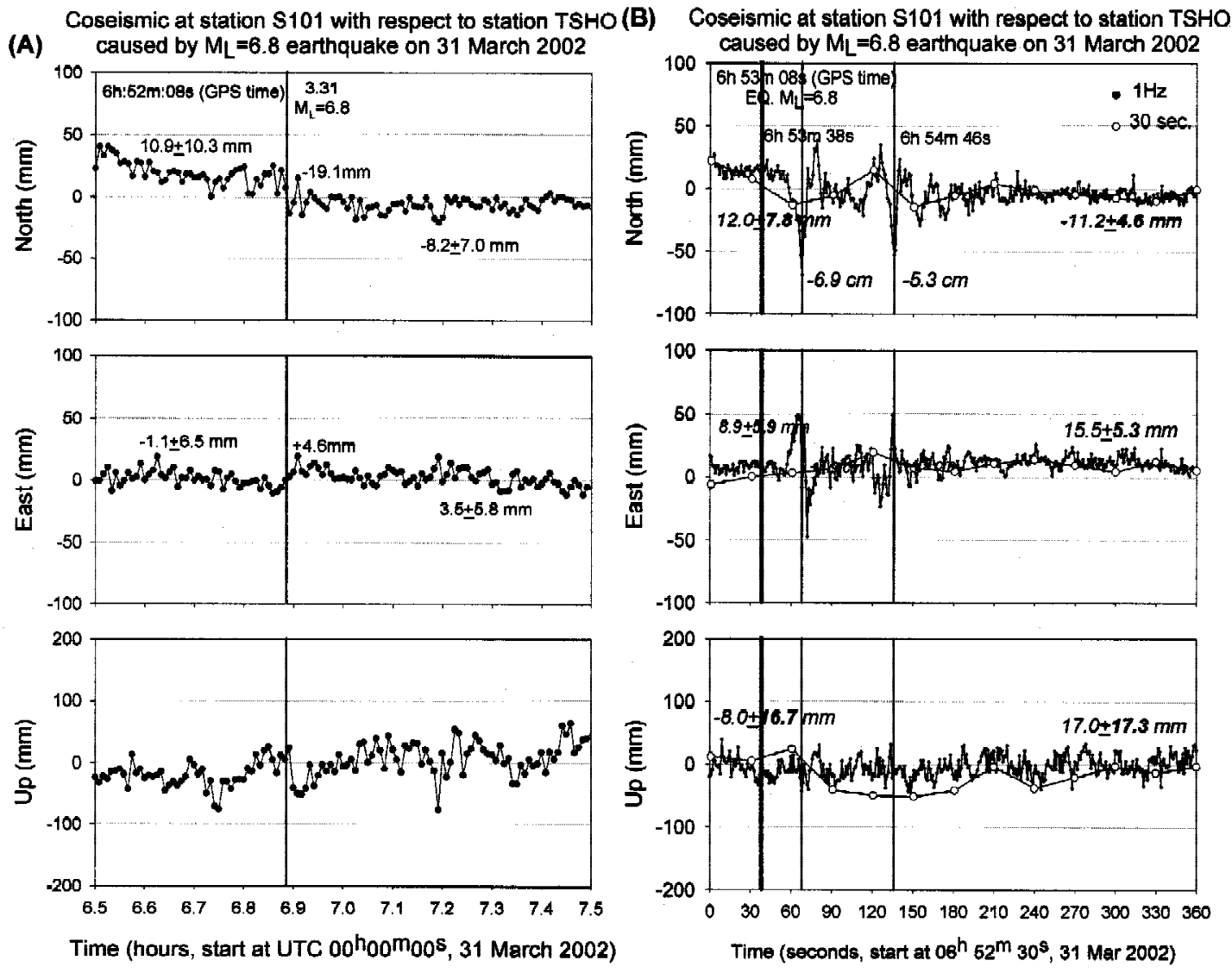

Fig. 5. Kinematic positioning results with 30 second sampling rate (5a), and with 1 second sampling rate (5b) in baseline S101-TSHO on 31 March 2002 (DoY, 090). In Fig. 5a, the abscissa presents the time series (units in hours), and the ordinate expresses the kinematic positioning results (units in millimeter). In Fig. 5b, the abscissa indicates the time series (units in seconds), and the ordinate represents the kinematic positioning results (units in millimeter).

2. The instantaneous ground motion can be detected using the kinematic positioning results in the 331 earthquake.

3. Considering the effects of the arrival time and the seismic ground motion, the further the relative station, the more detailed ground motion can be obtained.

4. For the kinematic positioning results, the coseismic deformations can be computed from the 30 -second sampling rate or 1 -second sampling rate data, with only a few millimeters difference between the results from the daily solutions. 


\section{REFERENCES}

Bock, Y., R. M. Nikolaidis, P. J. de Jonge, and M. Bevis, 2000: Instantaneous geodetic positioning at medium distances with the Global Positioning System. J. Geophys. Res., 105, 28223-28253.

Central Weather Bureau (CWB) earthquake report, Taiwan, 2002: web page http://www.cwb. gov.tw/V4/index.htm.

Chien, T. L., 1999: The 921 earthquake emergency measures. In: Proc. Inter. Workshop on the Chi-Chi, Taiwan earthquake of September 21, 1999, Taichung, 10, 1-10.

Chen H. Y., L. C. Kuo, W. S. Chung, and S. B. Yu, 2003a: Using Quasi Ionosphere-Free PostProcessing Algorithm on The Medium-Range Kinematic High Accuracy GPS Relative Positioning. Wuhan Univ. J. Natural Sci., 8, 610-618.

Chen H. Y., L. C. Kuo, W. S. Chung, and S. B. Yu, 2003b: Seismic ground motion observed by the Taiwan continuous GPS array - in the case of 31 March 2002 Hualien earthquake. Proc. Inter. Symp. GPS/GNSS, Tokyo.

Han S., and C. Rizos, 2000: GPS multipath mitigation using FIR filters. Survey Rev., 35, 487 498.

Kuo L. C., 2001: High Precision GPS Surveying for Crustal Deformation Study, PhD dissertation, Dept. Civil Engin., Nat. Chiao-Tung Univ., Taiwan, 201p. (in Chinese)

Mervart L., 1995: Ambiguity Resolution Techniques in Geodetic and Geodynamic Application of the Global Positioning System, PhD Thesis, Geodätisch-geophysikalische Arbeiten in der Schweiz, Band 53.

Remondi, B. W., and G. R. Brown, 2000: Triple differencing with Kalman filtering: Make it work. GPS Solution, 3, 58-64.

Rizos C., S. Han, L. Ge, H. Y. Chen, Y. Hatanaka, and K. Abe, 2000: Low-cost densification of permanent GPS networks for natural hazard mitigation: first tests on GSI's Geonet network. Earth Planet. Sci., 52, 867-871.

Yu S. B., H. Y. Chen, and L. C. Kuo, 1997: Velocity field of GPS stations in the Taiwan area. Tectonophys., 274, 41-59. 